\title{
Numerical simulations of the life cycle of dust in forming and evolving disk galaxies
}

\author{
Kenji Bekki* \\ ICRAR at University of Western Australia \\ E-mail: bekki@cyllene.uwa.edu.au
}

\begin{abstract}
We present the first self-consistent numerical simulations of the time evolution of dust properties, molecular hydrogen $\left(\mathrm{H}_{2}\right)$ contents, and star formation histories in forming disk galaxies. We focus mainly on the formation of disk galaxies with total masses ranging from $10^{10} M_{\odot}$ to $10^{12}$ $M_{\odot}$ in a Cold Dark Matter (CDM) cosmology with the latest WMAP 7-year results. The principle results are as follows. The star formation histories of disk galaxies can be regulated by the time evolution of interstellar dust, mainly because the formation rates of $\mathrm{H}_{2}$ can be controlled by dust properties. More massive disk galaxies with higher stellar surface densities are likely to have smaller dust-to-star mass ratios $\left(s_{\mathrm{d}}\right)$. The observed correlation between PAH-to-dust mass ratios $\left(q_{\mathrm{PAH}}\right)$ and gas-phase oxygen abundances $\left(A_{\mathrm{O}} \equiv 12+\log (\mathrm{O} / \mathrm{H})\right)$ can be reproduced reasonably well in the present models. The disks show negative radial gradients (i.e., larger in inner regions) of $\mathrm{H}_{2}$ fraction $\left(f_{\mathrm{H}_{2}}\right), q_{\mathrm{PAH}}$, dust-to-gas ratio $(D)$, and $A_{\mathrm{O}}$ and these gradients evolve with time. More massive disk galaxies are more likely to have higher $D, q_{\mathrm{PAH}}$, and $f_{\mathrm{H}_{2}}$. Early-type E/S0 galaxies formed by major galaxy merging can have lower $s_{\mathrm{d}}$ than isolated late-type disk galaxies.
\end{abstract}

The Life Cycle of Dust in the Universe: Observations, Theory, and Laboratory Experiments 18-22 November, 2013

Taipei, Taiwan

* Speaker. 


\section{From one-zone models to $\mathrm{N}$-body simulations}

Recent observational studies by infrared space telescopes have revealed physical properties of dust, their spatial distributions, and their correlations with their host galaxy properties in nearby and distant galaxies (e.g., [1-3]). For example, [2] have derived the detailed 2D maps of dustto-gas-ratio $(D)$, dust temperature, and PAH fraction in the Large Magellanic Cloud (LMC). [1] have investigated the total dust mass, the mass fraction of the dust contributed by PAHs, and the correlation between gas-phase oxygen abundance $\left(A_{\mathrm{O}}=12+\log (\mathrm{O} / \mathrm{H})\right)$ and $D$ for 65 nearby galaxies and found that the average PAH fraction in galaxies with $A_{\mathrm{O}}>8.1$ is $3.55 \%$. [3] have found not only a significant spread in the dust-to-stellar-mass ratio between late-type spirals but also the dependences of the ratios on galaxy dynamical and morphological properties (e.g., surface mass densities).

The majority of previous theoretical studies of dust formation and evolution in galaxies are based on one-zone (or multi-zone) chemical evolution models (e.g., [4-7]). Although such previous models provided theoretical explanations for a number of key observational results on dust properties in galaxies (e.g., $A_{\mathrm{O}}-D$ relation), they did not allow astronomers to discuss the spatial distributions of dust properties and their correlations with other galaxy properties (e.g., Hubble morphological types). Therefore, it is largely unclear in these previous studies what physical mechanisms are responsible for the observed spatial distributions of dust properties in galaxies.

In this paper, we present our new N-body/hydrodynamical simulation code by which we can investigate the spatial distributions of dust properties in galaxies as well as the dependences of mean dust properties on galaxy properties. We mainly show the results of our simulations on disk galaxy formation in a CDM cosmology with the latest WMAP 7-year results on the cosmological parameters. The simulations properly address the life cycle of dust during disk galaxy formation via hierarchical merging of subgalactic clumps from $z \sim 16$ to $z=0$. We mainly investigate (i) correlations between gas mass ratios and dust-to-star mass ratios, (ii) dependences of the PAH mass fractions $\left(q_{\mathrm{PAH}}\right)$ on $A_{\mathrm{O}}$, and (iii) radial gradients of $D$ in disk galaxies at $z=0$. The details of the new simulation code that can be run on GPU (graphic processing unit) clusters are given in [8]. Therefore, we here briefly describe the present model below.

\section{The model}

We assume that a disk galaxy can form from a homogeneous, rigidly rotating gas sphere on which density fluctuations consistent with a CDM power spectrum are superimposed. We use the COSMICS software [9] in order to generate the CDM density fluctuations in each model. The initial total masses of disk galaxies (including both dark and baryonic matter) are free parameters ranging from $10^{10} M_{\odot}$ to $10^{12} M_{\odot}$. The initial spin parameter is set to be $0.02,0.04,0.06$, and 0.1 . We start numerical simulations of disk galaxy formation at $z \sim 16$ and thereby investigate the final dust properties of the simulated disk galaxies at $z=0$. Star formation rates are estimated from local $\mathrm{H}_{2}$ densities of gas clouds and supernova feedback effects both from SNII and SNIa are included self-consistently. The total number of particles per simulation is $\sim 10^{6}$ and the gravitational softening length is $93 \mathrm{pc}$ for baryonic matter and $930 \mathrm{pc}$ for dark matter in the present simulations. Dust is assumed to be produced both by AGB stars and by supernovae and the dust yields used in 


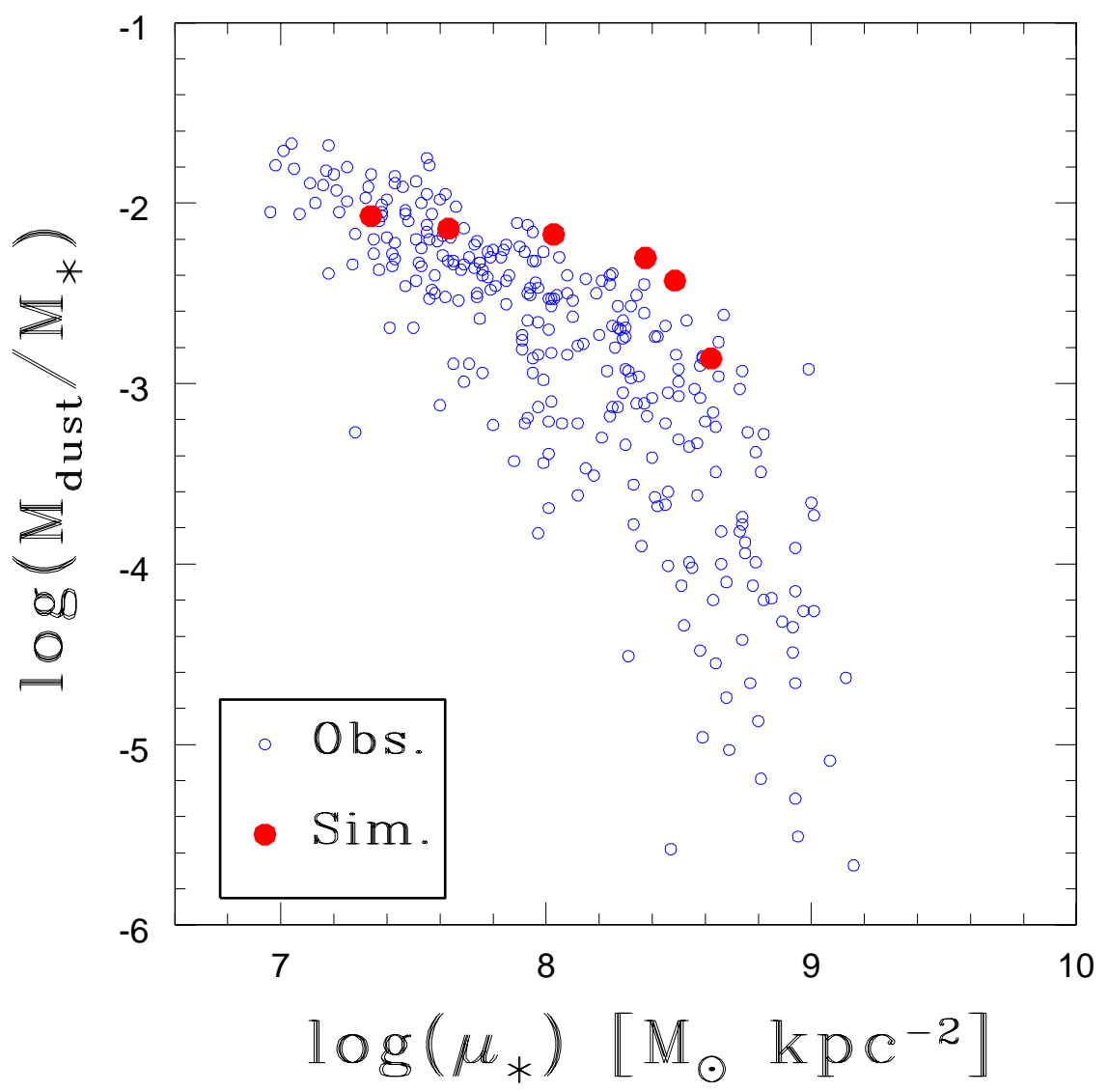

Figure 1: The observed (blue) and simulated (red) dust scaling relation. The total dust mass, stellar mass, and mean stellar surface mass density in a galaxy are denoted by $M_{\mathrm{dust}}, M_{*}$, and $\mu_{*}$, respectively. The dustto-star mass ratio is larger for galaxies with lower $\mu_{*}$ both for observations and simulations (six disk galaxies with different disk masses yet a fixed spin parameter of 0.06). However, the simulated dust scaling relation is shallower than the observed one. Furthermore, the observed galaxies with very low dust-to-star mass ratios $\left(s_{\mathrm{d}}<-3\right)$ cannot be well reproduced by the present disk galaxy formation models. These low- $s_{\mathrm{d}}$ galaxies are suggested to have experienced major merging in their histories ([8]).

this study are exactly the same as those adopted in [8]. We compare the present simulations results with the latest observational ones derived by Herschel and Spitzer telescopes (e.g., [3]). The star formation histories of disk galaxies can be regulated by the time evolution of interstellar dust, mainly because the formation rates of $\mathrm{H}_{2}$ can be controlled by dust properties. We however do not discuss this important issue in the present study (see [8] for the details of this issue).

\section{Results}

Fig. 1 shows the dust-to-star mass ratios $\left(s_{\mathrm{d}}=M_{\text {dust }} / M_{*}\right)$ as a function of mean stellar mass densities $\left(\mu_{*}\right)$ in the simulated disk galaxies with different initial masses at $z=0$. The more massive galaxies can have higher $\mu_{*}$ in the present study. Therefore, Fig. 1 demonstrates that more massive galaxies with larger $\mu_{*}$ can have smaller $s_{\mathrm{d}}$, which is qualitatively consistent with the observational 


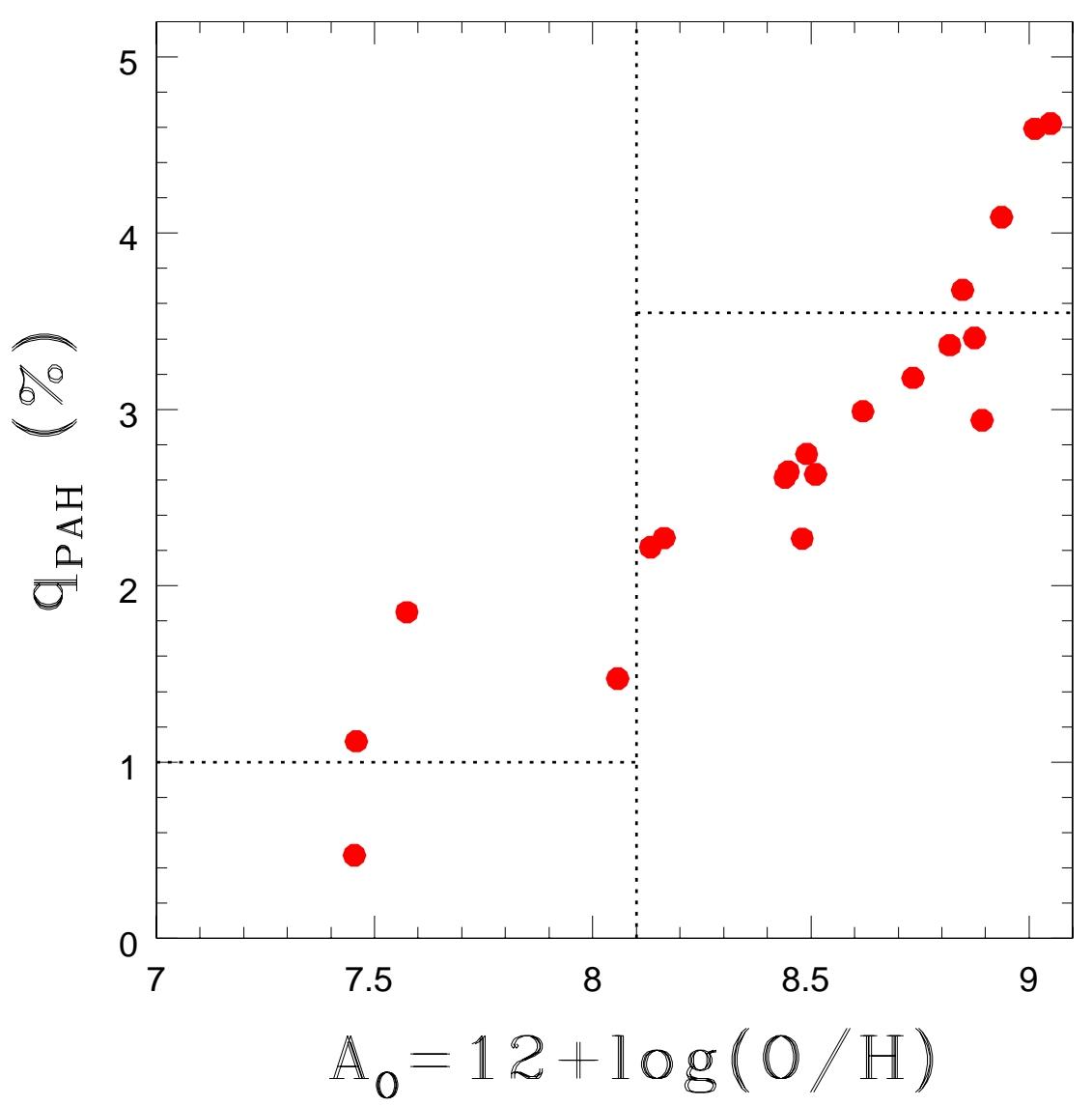

Figure 2: The PAH-to-dust mass ratios $\left(q_{\mathrm{PAH}}\right)$ of simulated disk galaxies as a function of their mean gasphase abundances $\left(A_{\mathrm{O}}\right)$. All of the simulated disk galaxies with different initial spin parameters and masses are shown in this figure. This result can be compared with the recent observational results by [1]. The horizontal dotted lines are the mean $q_{\mathrm{PAH}}$ for dwarfs and giant galaxies. The vertical dotted line is a threshold $A_{\mathrm{O}}$ below which $q_{\mathrm{PAH}}$ is observed to be very low [1]. The present simulations can reproduce well the observed correlation between $q_{\mathrm{PAH}}$ and $A_{\mathrm{O}}$.

results. However, the simulated slope is shallower than the observed one, which possibly means that the present dust recycling model (or $\mathrm{H}_{2}$ formation model) could have some problems. The present simulations also show that more massive disk galaxies at $z=0$ can have smaller gas mass fractions and smaller $s_{\mathrm{d}}$, which is consistent with observations [3]. Our simulations, however, can not explain the observed very high $s_{\mathrm{d}}$ (almost 0.02 ) in low-mass, and very gas-rich dwarf disk galaxies. The observed low $s_{\mathrm{d}}$ can be closely associated with past major merger events that form E/S0 galaxies [8].

Fig. 2 shows that more massive, more metal-rich (i.e., larger $A_{\mathrm{O}}$ ) disk galaxies are likely to have higher $q_{\mathrm{PAH}}$, which is qualitatively consistent with recent observations by [1]. In the present dust model, about $5 \%$ of carbon from carbon-rich AGB stars is assumed to become PAH dust. Low-mass disk galaxies show suppressed star formation due to strong supernova feedback effects so that chemical evolution can proceed very slowly. As a result of this, low-mass disk galaxies can 


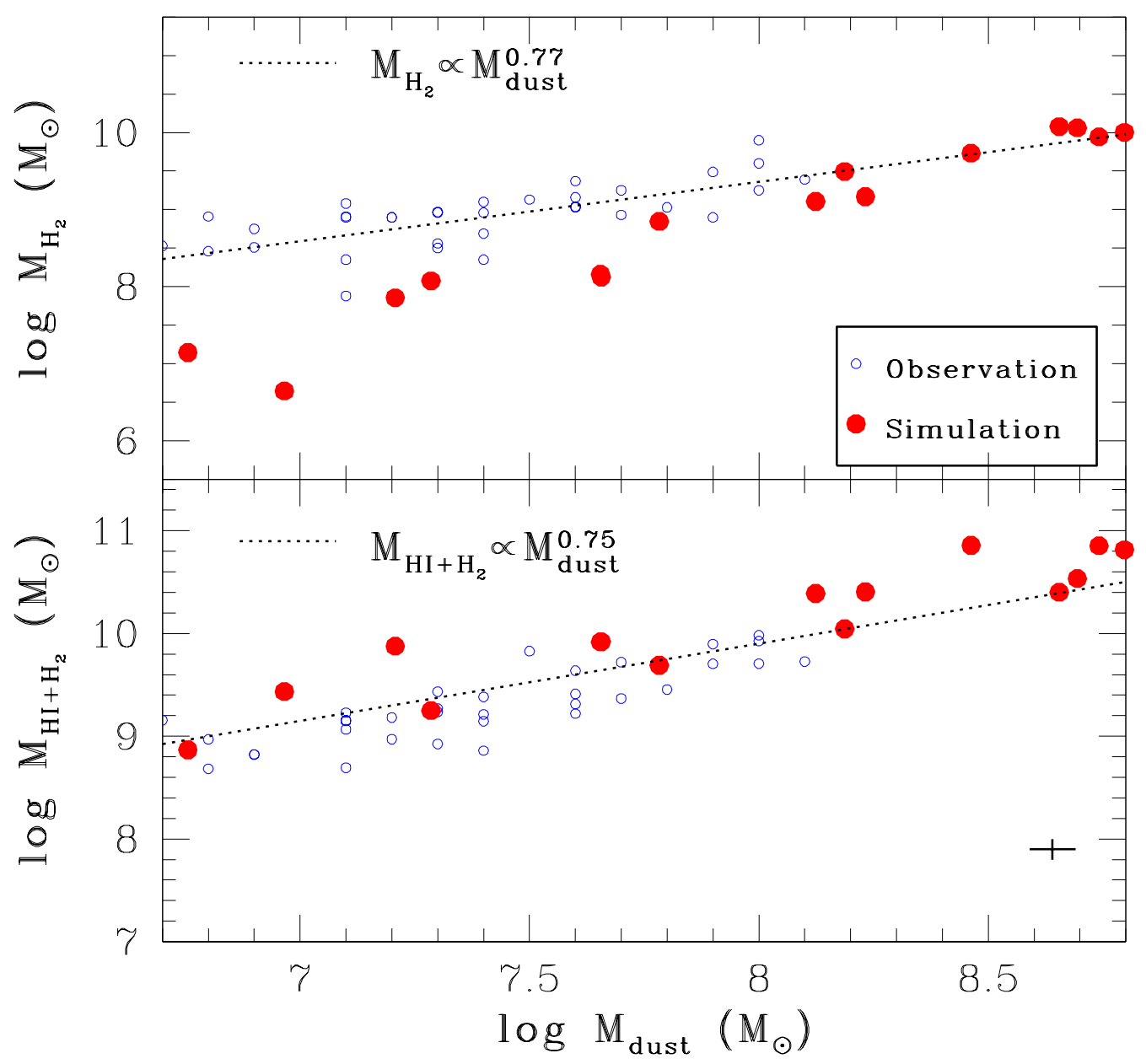

Figure 3: The dependences of total $\mathrm{H}_{2}$ masses $\left(M_{\mathrm{H}_{2}}\right.$, upper $)$ and total gas masses $\left(M_{\mathrm{HI}}+M_{\mathrm{H}_{2}}\right)$ on total dust masses $M_{\text {dust }}$ for observations (blue) and simulations (red). The dotted lines are the observed scaling relations from [10].

have a smaller number of C-rich AGB stars and thus have low $q_{\mathrm{PAH}}$ in the present study. Therefore, the present simulations can provide a physical origin for the observed $q_{\mathrm{PAH}}-A_{\mathrm{O}}$ correlation.

Fig. 3 shows that the simulated correlation between dust masses and total gas masses is reasonably consistent with the observed ones. The observed scaling relation $\left(M_{\mathrm{HI}+\mathrm{H}_{2}} \propto M_{\text {dust }}^{0.75}\right)$ derived by [10] can be reproduced by the present model in a quantitatively manner for a wide range of dust mass. However, the simulated correlation between dust masses and total $\mathrm{H}_{2}$ masses does not match so well with the observed one. In particular, galaxies with lower dust masses deviate significantly from the observed relation (smaller $\mathrm{H}_{2}$ mass for a given dust mass). This means either that the present dust and $\mathrm{H}_{2}$ modeling can significantly underestimate the total $\mathrm{H}_{2}$ masses for low-mass disk galaxies or that dust mass can be over-produced owing to the shorter dust growth timescale in low-mass disk galaxies. The total number of the observed galaxies with $M_{\text {dust }} \leq 10^{7} M_{\odot}$ is still small in this figure so that we can not make a robust conclusion on this possible inconsistency between observations and simulations. The simulated disk galaxies show negative radial gradients of dust-to-gas ratios in all models. 


\section{Conclusions}

We have shown, for the first time, that the observed dust scaling relations can be reproduced reasonably well by disk galaxy formation models based on a CDM cosmology. However, there are a number of observations that can not be reproduced so well by the present models (e.g., the observed correlation between $\mathrm{H}_{2}$ and dust masses for galaxies with $M_{\text {dust }} \leq 10^{7} M_{\odot}$ ). This could be related to the adopted rather idealized model for dust accretion and destruction. We therefore need to incorporate a more realistic dust model (e.g., including effects of radiation pressure on dust) into N-body/hydrodynamical simulations with star formation in our future work. Also we need to separately model the evolution of gas and dust in our future works, because gas and dust can mutually influence their dynamics in galaxies.

\section{References}

[1] B. T. Draine, et al., Dust Masses, PAH Abundances, and Starlight Intensities in the SINGS Galaxy Sample , 2012, ApJ, 663, 866

[2] M. Meixner, et al. 2010, HERschel Inventory of The Agents of Galaxy Evolution (HERITAGE): The Large Magellanic Cloud dust, 2010, A\&A, 518, L71

[3] L. Cortese, et al., The dust scaling relations of the Herschel Reference Survey, 2012, A\&A, 540, 52

[4] E. Dwek, The Evolution of the Elemental Abundances in the Gas and Dust Phases of the Galaxy, 1998, ApJ, 501, 643

[5] U. Lisenfeld, A. Ferrara, Dust-to-Gas Ratio and Metal Abundance in Dwarf Galaxies, 1998, ApJ, 498,145

[6] H. Hirashita, Dust-to-Gas Ratio and Metallicity in Dwarf Galaxies, 1999, ApJ, 522, 220

[7] M. Edmunds, An elementary model for the dust cycle in galaxies, 2001, MNRAS, 328, 223

[8] K. Bekki, Coevolution of dust, gas and stars in galaxies - I. Spatial distributions and scaling-relations of dust and molecular hydrogen, 2013, MNRAS, 432, 2298

[9] E. Bertschinger, COSMICS: Cosmological Initial Conditions and Microwave Anisotropy Codes, 1995, arXiv:astro-ph/9506070

[10] E., Corbelli, et al., The Herschel Virgo Cluster Survey. X. The relationship between cold dust and molecular gas content in Virgo spirals , 2012, A\&A, 542, 32 